\title{
Factors Associated With Changes in Magnesium Levels in Asymptomatic Neonates: A Longitudinal Analysis
}

\author{
Yogesh Mehta, ${ }^{1, *}$ Charudatta Shitole, ${ }^{1}$ and Maninder Singh Setia ${ }^{2}$ \\ ${ }^{1}$ Department of Pediatrics, Dr. L H Hiranandani Hospital, Mumbai, India \\ ${ }^{2}$ Dr. L H Hiranandani Hospital, Mumbai, India \\ ${ }^{*}$ Corresponding author: Yogesh Mehta, Department of Pediatrics, Dr. L H Hiranandani Hospital, Hillside Avenue, Hiranandani Gardens, Mumbai, India. Tel: +91-2225763333, \\ Fax: +91-2225763344, E-mail: yogesh.mehta@hiranandanihospital.org
}

Received 2015 June 9; Revised 2015 October 26; Accepted 2015 October 31

\begin{abstract}
Background: Neonates and infants with hypomagnesemia present with seizures and psychomotor delay.

Objectives: The present study evaluated the changes in magnesium $(\mathrm{Mg})$ levels and factors associated with these in the first three days of life.

Materials and Methods: We monitored 50 clinically asymptomatic neonates; they were not given any magnesium supplements even if they had hypomagnesemia at baseline. The variables analysed were: serum Mg; gestational age; birth weight; length; and the ponderal index. We used random effects (RE) models for longitudinal analysis of these data.

Results: The mean standard deviation(SD) gestational age was 36.3(3.6) weeks and the mean(SD) weight was 2604.2 (754.4) grams. About $31 \%$ of the neonates had hypomagnesemia $(<1.6 \mathrm{mg} / \mathrm{dL}$ ) on day one; however, all had normal magnesium levels by day three of life ( $\mathrm{P}<$ 0.001). At birth, after adjusting for intrauterine growth retardation status (IUGR), serum Mg levels were lower by $0.0097 \mathrm{mg} / \mathrm{dL}$ ( $95 \% \mathrm{CI}$ : -0.019 to -0.0003$)$ per 100 grams increase in weight of the neonate. After adjusting for IUGR status, the mean increase in the serum Mg levels was $0.14 \mathrm{mg} / \mathrm{dL}$ (95\% confidence intervals [CI]: 0.10 to 0.18 ) per day. The per-day increase in magnesium levels was significantly higher in low birth weight babies (0.10, $95 \%$ CI: 0.01 to 0.18$)$ compared with normal birth weight babies.

Conclusions: Asymptomatic neonates may have a high prevalence of hypomagnesemia; however, the levels become normal without any magnesium supplementation. Even though regular monitoring of magnesium levels is useful, no supplements are required-particularly in clinically asymptomatic neonates.
\end{abstract}

Keywords: Magnesium, Supplementation, Hypomagnesemia

\section{Background}

Magnesium (Mg), an abundant cation in the human body, plays an important role in multiple physiological functions (1). It is important for enzyme activation, muscular contraction and relaxation, neuronal activity and neurotransmitter release, and cardiovascular activities (such as cardiac function, vasomotor tone) (1, 2). Hypomagnesemia in neonates often manifests as neonatal seizures and psychomotor delay $(3,4)$. It may be a congenital condition and researchers have reported that it is associated with therapeutic hypothermia in neonates (3, $5)$. It is often seen in children born to women with gestational diabetes $(6,7)$.

The reports on changes in magnesium levels after birth have presented mixed findings. For instance, some authors did not find any changes in the levels of magnesium in the first week of life (8), others have stated that levels rise initially and subsequently stabilize (9). Furthermore, there is little consensus on addressing hypomagnesemia in neonates. While some researchers have used magnesium supplements for hypomagnesemia (10), others found that low Mg levels became normal even in the absence of treatment (11). The need for more studies to understand magnesium homeostasis has also been highlighted by researchers (9).

\section{Objectives}

Thus, we designed the present longitudinal study to assess the changes in magnesium levels in neonates in the first three days of life and the factors associated with change in these neonates.

\section{Materials and Methods}

The present study is a longitudinal analysis of secondary clinical data from 50 clinically asymptomatic newborn children over a period of three months. It was conducted at a private tertiary care hospital in Mumbai, India. It is a sub-specialty hospital in suburban Mumbai and includes facilities for intensive care (adult, medical, surgical, and neonatal). Data were collected from clinical charts of patients. We used the following clinico-demographic variables for the present analysis: serum magnesium levels on

Copyright (C) 2016, Growth \& Development Research Center. This is an open-access article distributed under the terms of the Creative Commons Attribution-NonCommercial 4.0 International License (http://creativecommons.org/licenses/by-nc/4.0/) which permits copy and redistribute the material just in noncommercial usages, provided the original work is properly cited. 
days one and three of life; gestational age; birth weight; length of the neonate; and the ponderal index. We created the following variables: term or preterm babies (cut-off of 37 weeks); birth weight classified as less than 2,500 grams and more than or equal to 2,500 grams; appropriate for gestational age and small for gestational age (SGA); and a ponderal index (PI) up to 2.0 and a PI greater than 2.0. Since, asymptomatic neonates are usually discharged after three days; we had clinical data only in the first three days of life. The blood test was done on cord blood (collected for blood group) on day one of life and on day three (wherever available). As indicated in the clinical charts, these neonates were monitored closely and were not given any magnesium supplements.

Data were entered in the MsExcel (Microsoft Corporation, USA) and converted to Stata Version 13 (StataCorp, College Station, Texas, USA) for analysis. We calculated the means and standard deviations for the continuous variables and proportions for categorical variables. The means were compared using the t-test and the proportions were compared using the chi square test and Fisher's exact test. We used linear regression models for multivariate analysis. We then used the random effects models for linear outcomes. These models are useful for modeling of time dependent variables (such as magnesium levels over time) and estimate within-individual and between-individual ef- fects; thus, they also account for correlation for repeated outcomes in the same individual. The main outcome variable was the serum magnesium levels, in the univariate and multivariate models. We used the Akaike information criteria to assess the fit of these models. Since it was a secondary data analysis, we had to use available clinical data. Hence, we estimated the power of our study. The power to estimate the mean $\mathrm{Mg}$ levels of 2.16 on day three compared with 1.76 on day one with an alpha of 0.05 and SD of change of 0.42 was $>99 \%$.The study was approved for secondary data analysis by the ethics committee at the Dr. LH Hiranadani Hospital, Mumbai, India.

\section{Results}

The mean (SD) gestational age of these 50 neonates was 36.3 (3.6) weeks and the mean (SD) weight was 2604.2 (754.4) grams. About $16 \%$ of the neonates were small for gestational age and $40 \%$ were preterm births. About $32 \%$ of the neonates had a weight of less than 2500 grams and $18 \%$ had a PI of less than 2.0 at birth. In our sample, we recorded two deaths by the third day; there was no significant difference in the mean magnesium levels between the neonates who had died and those who were alive (Table 1). In fact both the neonates who had died had normal magnesium levels on day one of birth.

\begin{tabular}{|c|c|c|c|c|c|}
\hline \multirow[t]{2}{*}{ Characteristics } & \multicolumn{2}{|c|}{ Day One } & \multicolumn{2}{|c|}{ Day Three } & \multirow[t]{2}{*}{ PValue $^{\mathrm{b}}$} \\
\hline & $\mathbf{n}$ & Mean (SD) & $\mathbf{n}$ & Mean (SD) & \\
\hline All & 49 & $1.76(0.20)$ & 47 & $2.16(0.44)$ & $<0.001$ \\
\hline \multicolumn{6}{|l|}{ IUGR status } \\
\hline SGA & 8 & $1.89(0.19)$ & 6 & $2.52(0.61)$ & 0.02 \\
\hline AGA & 41 & $1.73(0.20)$ & 41 & $2.10(0.39)$ & $<0.001$ \\
\hline P Value & & 0.04 & & 0.03 & \\
\hline \multicolumn{6}{|c|}{ Gestational age status } \\
\hline Preterm & 20 & $1.83(0.15)$ & 17 & $2.37(0.56)$ & $<0.001$ \\
\hline Full term & 29 & $1.70(0.22)$ & 30 & $2.04(0.30)$ & $<0.001$ \\
\hline P Value & & 0.03 & & 0.01 & \\
\hline \multicolumn{6}{|l|}{ Birth weight,g } \\
\hline Upto 2500 & 16 & $1.89(0.13)$ & 13 & $2.51(0.59)$ & $<0.001$ \\
\hline$>2501$ & 33 & $1.69(0.20)$ & 34 & $2.02(0.30)$ & $<0.001$ \\
\hline P Value & & $<0.001$ & & $<0.001$ & \\
\hline \multicolumn{6}{|l|}{ Ponderal index } \\
\hline $0-2.00$ & 9 & $1.89(0.17)$ & 6 & $2.42(0.70)$ & 0.04 \\
\hline$>2.01$ & 40 & $1.73(0.20)$ & 41 & $2.12(0.39)$ & $<0.001$ \\
\hline P Value & & 0.03 & & 0.12 & \\
\hline \multicolumn{6}{|c|}{ Gestation diabetes mellitus in the mother } \\
\hline Yes & 4 & $1.89(0.31)$ & 4 & $2.13(0.30)$ & 0.29 \\
\hline No & 45 & $1.75(0.19)$ & 43 & $2.16(0.45)$ & $<0.001$ \\
\hline P Value & & 0.19 & & 0.91 & \\
\hline \multicolumn{6}{|l|}{ Death } \\
\hline Yes & 2 & $1.91(0.01)$ & NA & NA & NA \\
\hline No & 46 & $1.75(0.20)$ & NA & NA & NA \\
\hline P Value & & 0.27 & & NA & \\
\hline
\end{tabular}

Abbreviations: AGA, Appropriate for Gestational Age; IUGR, Intrauterine Growth Retardation; NA, not available; SGA, Small for Gestational Age.

${ }^{\mathrm{a}}$ The numbers may not add up due to missing data.

$\mathrm{b}_{\text {The }} \mathrm{P}$ value denotes the differences between the means at baseline and third day. 
About 31\% of the neonates had low serum magnesium levels on day one and none of them had lower magnesium levels at day three; the reduction was statistically significant $(\mathrm{P}<0.001)$. The mean $(\mathrm{SD})$ of serum magnesium at birth was $1.76(0.20) \mathrm{mg} / \mathrm{dL}$; the levels had increased significantly by day three $(2.16$ [0.44], $\mathrm{P}<0.001)$. We found that the mean serum magnesium levels were significantly higher in SGA neonates compared with appropriate for gestational age neonates, both at baseline and on day three. Similarly, the mean magnesium levels were significantly higher in preterm children compared with those born at full term. The mean magnesium levels were significantly higher in low birth weight infants (weight $<2500$ grams) at day one as well as day three (Table 1). Although, the mean magnesium levels were significant higher in neonates with a low Ponderal Index at birth, the difference was not statistically significant on the third day. The mean magnesium levels were not significantly different in neonates born to mothers with gestational diabetes mellitus compared with others. We have provided detail means and SDs across various categories on day one and day three in Table 1. After adjusting for intrauterine growth retardation status (IUGR), we found that serum magnesium levels were lower by $0.0097 \mathrm{~g} /$ dL (95\% confidence intervals [CI]: -0.019 to -0.0003) per 100 grams increase in the birth weight of the neonate at baseline. The levels were also lower by $0.031 \mathrm{~g} / \mathrm{dL}$ (95\% CI: -0.053 to -0.009) per 100 grams increase in the weight on the third day.

In the random effects linear models, we found that across all four different types of models, there was a significant increase in the mean levels of serum magnesium per day of follow-up (Table 2). Furthermore, the multivariate models also showed that the interaction between low birth weight and per day increase was significant. Thus, the per-day increase in magnesium levels was significantly higher in low birth weight babies $(0.10 \mathrm{~g} / \mathrm{dL}$, 95\% CI: 0.01 to 0.18) compared with normal birth weight babies. Gestational diabetes was not associated with hypomagnesemia in any of the models. We have presented the estimates and the confidence intervals for univariate and multivariate models in Table 2.

Table 2. Table Showing the Estimates and 95\% Confidence Intervals (CI) From Random Effects Models in 50 Neonates, Mumbai, India

\begin{tabular}{lccccc}
\hline & Unadjusted Models & \multicolumn{2}{c}{ Adjusted Models } \\
\cline { 2 - 6 } & Estimate $(\mathbf{9 5} \% \mathbf{C I})$ & Estimate $(\mathbf{9 5} \% \mathbf{C I})$ & Estimate $(\mathbf{9 5} \% \mathbf{C I})$ & Estimate $(\mathbf{9 5} \% \mathbf{C I})$ & Estimate (95\% CI) \\
\hline Gestation age status & & & & NA & NA \\
Preterm & $0.21(0.05,0.36)^{\mathrm{a}}$ & $0.25(0.11,0.39)^{\mathrm{a}}$ & $\mathrm{NA}$ & $\mathrm{NA}$ & NA \\
Term & Reference & Reference & NA & NA
\end{tabular}

\section{IUGR status}

\begin{tabular}{|c|c|c|c|c|c|}
\hline SGA & $0.24(0.03,0.46)^{\mathrm{a}}$ & NA & $0.27(0.07,0.47)^{\mathrm{a}}$ & NA & NA \\
\hline AGA & Reference & NA & Reference & NA & NA \\
\hline \multicolumn{6}{|c|}{ Birth weight (grams) } \\
\hline Up to 2500 & $0.31(0.15,0.47)^{\mathrm{a}}$ & NA & NA & $0.20(0.02,0.37)^{\mathrm{a}}$ & NA \\
\hline$>2501$ & Reference & NA & NA & Reference & NA \\
\hline
\end{tabular}

\section{Ponderal index}

\begin{tabular}{|c|c|c|c|c|c|}
\hline $0-2.00$ & $0.17(-0.04,0.38)$ & NA & NA & NA & $0.21(0.02,0.41)^{\mathrm{a}}$ \\
\hline$>2.01$ & Reference & NA & NA & NA & Reference \\
\hline \multicolumn{6}{|l|}{ Gestational diabetes } \\
\hline Yes & $0.06(-0.22,0.34)$ & $0.16(-0.09,0.41)$ & $0.03(-0.23,0.28)$ & $0.07(-0.14,0.28)$ & $0.03(-0.22,0.29)$ \\
\hline No & Reference & Reference & Reference & Reference & Reference \\
\hline Day (per day increase) & $0.13(0.10,0.17)^{\mathrm{a}}$ & $0.14(0.10,0.18)^{\mathrm{a}}$ & $0.14(0.10,0.18)^{\mathrm{a}}$ & $0.11(0.06,0.16)^{\mathrm{a}}$ & $0.14(0.10,0.18)^{\mathrm{a}}$ \\
\hline \multicolumn{6}{|l|}{ Interaction terms } \\
\hline Low birth weight Day & NA & NA & NA & $0.10(0.01,0.18)$ & NA \\
\hline AIC & NA & 60.68 & 64.46 & 46.6 & 66.87 \\
\hline
\end{tabular}

Abbreviations: AGA, Appropriate for Gestational Age; AIC, Akaike Information Criteria; IUGR, Intrauterine Growth Retardation; NA, not available; SGA, Small for Gestational Age. $\mathrm{a}_{\mathrm{P}<0.05}$ 


\section{Discussion}

Even though about one-third of clinically asymptomatic neonates had low serum magnesium levels at birth, all of them had normal levels even without magnesium supplementation. Furthermore, the increase in levels was faster in the low birth weight neonates.

The prevalence of hypomagnesemia was relatively high in these asymptomatic neonates. Munoz and colleagues reported that hypomagnesemia was as high as $31.1 \%$ in neonates admitted to neonatal ICU; whereas Ahsan and co-workers found that the prevalence of hypomagnesemia was $21.8 \%$ in neonates with diarrhea $(12,13)$. In general, serum magnesium was higher in preterm neonates, those with a low birth weight, and those who were small for gestational age. Though hypomagnesemia has been reported in premature infants (12), others have reported that the magnesium levels were higher in neonates with a postconceptional age of less than 35 weeks (14). Furthermore, Ariceta and colleagues (14) also reported that gestational age was a significant contributory factor to the variations in serum magnesium levels. In contrast to the finding by Ariceta and colleagues, we found that after adjusting for other variables (such as IUGR status and gestational age), weight of the neonate is the single important factor associated with serum magnesium levels. Thus according to our study - higher the weight, lower the magnesium levels in neonates; this relation was maintained at baseline as well as the third day of life.

Serum Mg levels have been reported to be associated with birth variables and anthropometric parameters. We also found that there was an increase in the serum $\mathrm{Mg}$ levels each day after birth till the third day; this relation was significant even after adjusting for various anthropometric parameters. Bajpai and coworkers found that the mean (SD) value of serum Mg was 1.51 (0.12) mEq/L at birth (evaluated in cord blood); however, there were no significant changes in the first week of life (8). In contrast, Noone and colleagues reported magnesium levels increased and stabilized in infants whose birth weight was less than 1000 grams (9). Tsang and Oh found that mean Mg levels increased in both SGA and AGA infants, even though the mean levels remained low in SGA neonates compared with AGA neonates (11). In our study, we found that mean magnesium levels not only were higher in low birth weight neonates, but these levels also increased faster in them. Furthermore, the Mg levels remained significantly higher in SGA neonates compared with AGA neonates on the first and the third day.

Though, we had missing data at the first visit, RE models are useful in handling missing data. We would have also liked to have information on mother's magnesium levels; however, we did not have these values. In addition, we did not have information on serum calcium and other electrolytes; however, other researchers have also provided information only on magnesium levels (8). In spite of these limitations, the study provides useful insights into the changes of serum magnesium levels in neonates. Our study uses random effects models and adds a longitudinal depth to the clinical literature.

Thus, we would like to conclude that asymptomatic neonates may have a high prevalence of hypomagnesemia. However, our longitudinal analyses indicate that the levels increase within the first three days to normal values even in the absence of any magnesium supplementation. Thus, even though regular monitoring of magnesium levels is useful in neonates, supplements may not be necessary - particularly in asymptomatic neonates.

\section{Acknowledgments}

We would like to acknowledge Dr. S Chatterjee (CEO) for support and suggestions.

\section{References}

1. Swaminathan R. Magnesium metabolism and its disorders. Clin Biochem Rev. 2003;24(2):47-66. [PubMed: 18568054]

2. Altura BM, Altura BT. Role of magnesium in patho-physiological processes and the clinical utility of magnesium ion selective electrodes. Scand J Clin Lab Invest Suppl.1996;224:211-34. [PubMed: 8865438]

3. Ndiaye M, Dehanin T, Sow AD, Sene MS, Basse AM, Fall AL, et al [Familial congenital hypomagnesemia revealed by neonatal convulsions]. Arch Pediatr. 2013;20(11):1212-8. doi: 10.1016/j.arcped.2013.08.019. [PubMed: 24090669]

4. Visudhiphan P, Visudtibhan A, Chiemchanya S, Khongkhatithum C. Neonatal seizures and familial hypomagnesemia with secondary hypocalcemia. Pediatr Neurol. 2005;33(3):202-5. doi: 10.1016/j. pediatrneurol.2005.03.009. [PubMed:16139735]

5. Tocco NM, Hodge AE, Jones AA, Wispe JR, Valentine CJ. Neonatal therapeutic hypothermia-associated hypomagnesemia during parenteral nutrition therapy. Nutr Clin Pract. 2014;29(2):246-8. doi: $10.1177 / 0884533614522835$. [PubMed: 24549013]

6. Jones CW. Gestational diabetes and its impact on the neonate. Neonatal Netw. 2001;20(6):17-23. doi: 10.1891/0730-0832.20.6.17. [PubMed: 12144115]

7. Schwartz R, Teramo KA. Effects of diabetic pregnancy on the fetus and newborn. Semin Perinatol. 2000;24(2):120-35. [PubMed: 10805168]

8. Bajpai PC, Sugden D, Ramos A, Stern L. Serum magnesium levels in the newborn and older child. Arch Dis Child.1966;41(218):424-7. [PubMed: 21032445]

9. Noone D, Kieran E, Molloy EJ. Serum magnesium in the first week of life in extremely low birth weight infants. Neonatology. 2012;101(4):274-7. doi:10.1159/000335240. [PubMed: 22248691]

10. Maggioni A, Orzalesi M, Mimouni FB. Intravenous correction of neonatal hypomagnesemia: effect on ionized magnesium.JPediatr. 1998;132(4):652-5. [PubMed: 9580765]

11. Tsang RC. Serum Magnesium Levels in Low Birth Weight Infants Arch Pediatr Adolescent Med. 1970;120(1):44. doi: 10.1001/archpedi.1970.02100060078010.

12. Ahsan SK, al-Swoyan S, Hanif M, Ahmad M. Hypomagnesemia and clinical implications in children and neonates. Indian J Med Sci.1998;52(12):541-7. [PubMed:10327792]

13. Munoz R, Khilnani P, Ziegler J, Salem M, Catlin EA, Nussbaum S, et al. Ultrafilterable hypomagnesemia in neonates admitted to the neonatal intensive care unit. Crit Care Med.1994;22(5):815-20. [PubMed: 8181290]

14. Ariceta G, Rodriguez-Soriano J, Vallo A. Magnesium homeostasis in premature and full-term neonates. Pediatr Nephrol. 1995;9(4):423-7. [PubMed: 7577401] 